\title{
Exploring Psychological Vaccination for Potentially Traumatized Children
}

\section{Abstract}

Objectives: Traumatic events and associated stress due to ongoing war and displacement may have severe adverse effects on psychosocial development of children. Although more than half of traumatized children are expected to develop posttraumatic psychopathology, no effective prevention has been found. The present study aims to investigate if Crisis Intervention Program for Child and Adolescents (CIPCA) has protective effects on mental wellbeing of child survivors of the ISIS war and displacement in the Kurdistan Region of Iraq.

Method: Data were gathered two years after the displacement experience and application of CIPCA (a semistructured single one-hour group intervention that has been hypotheses to act as psychological vaccination against development of posttraumatic psychopathology. The study sample consisting of 51 children 32 girls and 19 boys, mean age 12 years), was cluster randomized immediately after displacement to CIPCA (23 children) and no CIPCA intervention (28 children). Behavior problems were estimated on the Child Behavior Checklist (CBCL) before randomisation and after one-year and 2-years follow-up.

Results: Significantly lower symptom score were found among children who had attended CIPCA than non-CIPCA concerning Internalizing scores $(0.63,7.40, \mathrm{t}=$ 2.099, $\mathrm{P}=0.049)$, Withdrawal $(0.0,2.5, \mathrm{t}=2.713, \mathrm{P}=0.014)$, PTSD index $(0.21$, $2.64, \mathrm{t}=2.264, \mathrm{P}=0.028)$ and Total Problem Sores $(1.82,13.07, \mathrm{t}=2.199, \mathrm{P}$ $=0.033$ ), consequently. No significant difference was observed according to Externalizing problems and other CBCL scores between children who had attend to CIPCA and not.

Conclusions: These findings pointed out that CIPCA may have protected children against developing psychological problems. We recommend carrying out more systematic studies to investigate the preventive effects of CIPCA on posttraumatic psychopathology.

Research Article
Volume 9 Issue 1 - 2018

Veysi Ceri'1* and Abdull baghi Ahmad ${ }^{2,3}$
${ }^{1}$ Senior Consultant in Child and Adolescent Psychiatry,
Marmara University, Turkey
${ }^{2}$ Associate Professor, Senior Consultant in Child and Adolescent
Psychiatry, Uppsala University, Sweden
${ }^{3}$ IACAPAP Ambassador, Founder of Child Mental Health \&
Investor of Metin Health House Duhok, Kurdistan Region, Iraq
*Corresponding author: Veysi CCeri, Senior Consultant in
Child and Adolescent Psychiatry, Marmara University, Pendik
Training and Research Hospital, Istanbul, Turkey,
Email: veysiceri@gmail.com
Received: February 07, 2018 | Published: February 15,
2018

Abbreviations: CIPCA: Crisis Intervention Program for Children and Adolescents; IDP: Internally Displaced People; ISIS: Islamic State in Iraq and Syria; KRI: Kurdistan region of Iraq; ToT: Training of Trainers

\section{Introduction}

Global population of forcibly displaced people has been reported to exceed 65 million in 2015 [1]. Although international community is highly aware of people who had passed the borders of their country and called as refugees, internally displaced people (IDP) are far more than refugees and not getting as much attention as refugees by the civilized world. As a consequence of collapsing of the Iraqi army against the Islamic State in Iraq and Syria (ISIS), hundreds of thousands of people fled to Kurdistan Region of Iraq (KRI) to escape being killed or become sex slaves. Children constitute major part of the survivors living in the camps or tents. Traumatic events are prevalent among child survivors of war $[2,3]$. Several studies show displaced children reporting high rates of exposure to severe traumatic events such as witnessing death or injury of someone and clashes or blasts [2-5]. Traumatic events and associated stress due to ongoing war and displacement may have severe adverse effects on psychosocial development of children [6] and lead emerging of depression, posttraumatic stress disorder and anxiety disorders [7,8]. High rates of other psychiatric symptoms than PTSD have also been showed among that displaced children have a ten times higher risk for developing PTSD [12]. Previous studies also indicate that PTSD would emerge among 9 to 45 percent of refugee children [2,12]. Despite strong evidences that traumatic events increase psychiatric morbidity such as PTSD and initial distress might be far greater than PTSD, there is no effective way to protect children from developing PTSD after traumatic exposure [13].

Vaccination is one of the greatest medical achievements of humankind that helped eradicating tens of epidemic childhood diseases which had lead death or disability of millions of children [14]. Accordingly, early psychological interventions have been hypothesized to reduce the psychological morbidity after traumatic events. In order to prevent problems, preventive programs have been stated to be proactive that must carry out before the onset of a disorder [15]. However, the blurry nature of psychiatric disorders does not allow a clear distinction between primary prevention (people do not have any symptom) and early intervention (people have mild symptoms) [16]. displaced children in numerous studies [4,9-11]. It is estimated 
However, It has been stated that the distinction between primary prevention and early intervention is not required in case of mental disorders because of the unclearness of the onset of the psychiatric disorders. Thus, any intervention that aims to prevent development of psychopathology among risky children could be named as psychological immunisation [16]. Although all of the interventions made by the mental health professionals to help their clients are forms of prevention [16], 'Psychological immunization' or 'Primary prevention', the concept that aiming to buffer children under risk against development of later psychopathology, has been proposed to be the "fourth mental health revolution" [17]. The literature on prevention programs and their effectiveness which assessed by the power of such programs in preventing development of disorder or reducing the incidence of later psychopathology is growing $[16,18,19]$. In one of such programs which aims to teach coping strategies based on cognitive behavioral techniques to children exposed to negative life events, results indicated reduction in depressive symptoms and externalizing problems [15]. Gilham et al. [20] suggested that psychological immunization could be achieved against depression 'by teaching cognitive and social skills to children as they enter puberty'. The efficacy of the depression preventing program was showed by a-) decline in previous symptoms and b-) less symptoms among children without initial symptoms over a 2-year follow-up [20]. The efficacy of the program also found to grow by time [20]. However, effectiveness of prevention interventions have not been proved in meta analysis [13] and further investigation is required [21]. Debriefing is also a psychological intervention that aims to reduce the psychological morbidity after traumatic exposure [22]. It consists of encouraging of survivor to express his emotion, support to process traumatic event and increase social connectedness [13]. It has been applied to child and adult survivors and witnessing numerous traumatic events and disasters with controversial results [13]. Although debriefing awaited to diminish posttraumatic distress and decrease the risk for development of later psychopathology, its protective effect has not been shown clearly [13]. It has been stated that primary prevention would be impossible in case of disorders that have not specific etiologic factors [16]. Although we do not have specific etiologic factors in most of psychiatric disorders, abundant number of evidences have showed link between traumatic events and later PTSD $[7,11,21]$. Several studies have been conducted to treat PTSD among refugee children. However, evidence thus far does not inform how to prevent PTSD in refugee children and only supports individual methods to treat PTSD [21]. Crisis Intervention Program for Children and Adolescents (CIPCA) has been recently developed to prevent posttraumatic psychopathology among displaced children and adolescents during the ISIS war in Iraq and Syria $[18,19]$. It is a well-structured, easily applied, child friendly intervention which mainly depends on interaction between children in a single one hour group session $[18,19]$. Any person who has high school education and experience of working with children can apply for CIPCA training. It has been developed to encourage children to spontaneously express their thoughts and feelings in the aftermath of a crisis situation. A trained group leader support children's expression and understanding of the ongoing events. Preliminary findings from a pilot project show promising results $[18,19]$. The lack of effective prevention for posttraumtic psychopathology during childhood makes it necessary to find out a well-defined crisis group intervention for children and adolescents. Based on updated knowledge, the CIPCA has been developed and applied on war displaced children in Iraqi Kurdistan. The present paper is aimed to investigate the effects of CIPCA on child behavior problems at a two-years followup after cluster randomisation to CIPCA and non-CIPCA groups.

\section{Marterial and Methods}

\section{Target population}

Two months after the attack of the Islamic State in Iraq and Syria (ISIS) on the Ezidi-dominated province Shingal in the Kurdistan Region of Iraq (KRI) in August 2014, 700000 thousands of the Internally Displaced People (IDP) were settled in the preliminary camps around the city of Duhok in the KRI, majority consisting of children. Multidisciplinary teams were trained at the Metin Health House (MHH) in the city of Duhok to provide psychosocial service to the IDPs. Majority of the IDPs were living in temporary camps, empty houses, such as schools, uncompleted buildings, uninhabited houses or in parks and in forests, protecting themselves from the heat of the sun which sometimes exceeded $40^{\circ} \mathrm{C}$. Common for all families, they had lost members either in ISIS captivity, or being killed or not found. Many young men had returned to participate in the war against ISIS. While adults were preoccupied with their traumatic experiences and worries, children often ended up outside adult care and attention without school or structured activities. Searching for loved ones was ongoing. Rumors of mass executions, sex slavery, mass rapes, and horror scenario spread about ISIS terror and violence were widespread. Extended families of three generations reunited when they found each other. The hierarchical social system began to re-establish itself as a protective survival strategy. No outbreaks of diseases had been reported.

\section{Crisis Intervention Program for Children and Adolescents (CIPCA)}

This intervention was developed at the MHH to prevent posttraumatic psychopathology among the IDP children and adolescents $[18,19]$. It is a cost-effective and time-saving onehour single group session of structured crisis intervention provided through Training of Trainers (ToT) to groups of 1030 children (6-11 years) or adolescents (12-18 years). After succeeding a course of 10 hours theory and 20 hours practical training, any professional working with children is provided with a certificate qualifying for 1.5 ECTS high school credits. Every group session is led by two trained professionals; one acting as group leader and the other is co-leader. The group leader follows the Crisis Expression Guidelines to support participating children and adolescents to spontaneously verbalize and express their negative thoughts and emotions after crises experience, and to replace them with positive thoughts. This stepwise semistructured interview is based on several well-known theories, such as catharsis [23], debriefing [13], group therapy [24], cognitive paradigm [25], systemic theory [26], attachment theory [27], salutogenesis [28], and posttraumatic growth [29]. The trained co-leader simultaneously uses the Crisis Screening Instrument to identify children showing any sign or symptom 
of psychological distress that is qualifying to further individual assessment. This instrument is derived from two widely used screening questionnaires with satisfactory validity and reliability in Kurdistan society [30,31]; Children showing positive screening are to be referred for individual assessment by a trained expert in using a Modified Family Map to identify risk and protecting factors in three generations in addition to examination of psychiatric status for planning of adequate management [32]. The CIPCA is hypothyzed to act as psychological vaccination against development of posttraumatic psychopathology $[18,19]$.

\section{The Process of CIPCA application}

Training of trainers produce certified CIPCA trainers who can plan to train Group leaders at any suitable time. The certified group leaders are competent to apply the group intervention whenever a crisis situation occurs where children and/or adolescents are involved.

\section{The procedure of CIPCA intervention}

First stage: Training of Trainers.

Second stage: Training of Group leaders.

Third stage: Application of <group Intervention.

Fourth stage: referring screened cases for individual psychiatric assessment.

Fifth stage: treatment of the diagnosed cases.

\section{Content of CIPCA intervention}

Two instruments are used during the one-hour single group session containing up to 30 children or adolescents: The Cisis Expression Guidelines is used by the group leader to consequently ask every child 12 Socratic questions. The Crisis Screening Instrument is used by the co-leader to observe and record the name of any child showing at least one of the listed 10 distress symptoms in order to refer the positive cases to an individual psychiatric evaluation for adequate treatment.

\section{Results}

This study sample, consisting of 51 children (32 girls and 19 boys, mean age 12 years), was cluster randomized two months after displacement to CIPCA intervention (23 children) and no CIPCA intervention (28 children). Behavior problems on the Child Behavior Checklist [33] were estimated before randomization and after one-year and 2-years follow-up. Data of this study were analyzed two years after the displacement experience and application of CIPCA. No significant difference are found between the CIPCA and the non-CIPCA groups concerning gender differences, except older age of the girls in CIPCA compared with non-CIPCA (mean age $=13.2,10.7$ years) $(\mathrm{t}=-2.398, \mathrm{p}=0.022)$. At two years follow-up, significantly lower symptom score were found among CIPCA than non-CIPCA concerning Withdrawal (0.0, $2.5, \mathrm{t}=2.713, \mathrm{P}=0.014)$, PTSD index $(0.21,2.64, \mathrm{t}=2.264, \mathrm{P}=$ $0.028)$, Total Problem Sores $(1.82,13.07, t=2.199, P=0.033)$, and Internalizing scores $(0.63,7.40, t=2.099, \mathrm{P}=0.049)$, consequently. Comparing of other subscale scores did not show significant differences between CIPCA and non CIPCA group.

\section{Discussion}

The findings of this study on internally displaced children in Kurdistan region of Iraq showed that a single session of CIPCA after displacement from ISIS war showed significantly lower CBCL symptom scores than children who did not receive CIPCA, particularly concerning trauma related symptoms. This might support CIPCA as protective against emerging of posttraumatic symptomatology, particularly but not exclusively PTSD. Although our findings do not figure out the mental wellbeing of children under risk immediately after intervention, children who attended to a single CIPCA session found to have fewer PTSD symptoms and internalizing problems than children who did not attend to CIPCA at the 2 year follow-up. While externalizing problems were not differing between children, total CBCL scores were also fewer among children who attended CIPCA intervention. Although we did not have data from the index CBCL scores to show mental wellbeing of children for the time of the CIPCA intervention, the difference in internalizing symptoms, total problem scores and PTSD index scores between intervention group and control groups at 2 year follow up could be interpreted as relief also. It has a great importance to be aware of the fact that mental health of refugee children is a complex problem which requires understanding of numerous factors; such as living environment, education, style of parenting, socioeconomic state of the family and hosting country etc., that have potential influence on psychosocial development of children [21]. Thus, an integrated approach that has been based on psychosocial interventions and consist of psycho-educational and clinical understanding is estimated to be effective [3]. CIPCA was developed by taking into account all mentioned factors. Thus, CIPCA is expected to be appropriate for prevention of posttraumatic psychopathology among children. Study findings also reveal efficiency of a single CIPCA intervention which was applied immediately after exposure to traumatic events. Although it is impossible to figure out if the improvement of children's symptoms in intervention group means the intervention has relieving effect or the intervention protected children against development of psychological symptoms as a primary prevention effect, the CIPCA intervention may exert positive effect in both mechanisms. Thus we propose that CIPCA may have relieved children who already had higher psychological symptoms and may protected children who had not developed posttraumatic symptoms yet. The mental wellbeing of child survivors of war and displacement is key area of clinical work and research to understand how the interplay between biological, psychological, social, and cultural processes determines variations between reactions of people in a wide spectrum [21]. It has been stated that pre-migratory traumatic events and post-migration hardships such as dealing with new life setting and acceptance by the new culture and reorganization of family life are key factors in understanding the risk and protective factors shaping the mental health of refugee children and might be point for preventive interventions: [21] Listening to children, clarification of situation, supporting and forming bonds of attachment as well as encouraging them to express their feelings and emotions have been pointed out as the main tools of interventions following traumatic events [34]. CIPCA, which is based on numerous theories, such as catharsis [23], debriefing [13], group therapy 
[24], cognitive paradigm [25], systemic theory [26], attachment theory [27] and salutogenesis [28]. Furthermore, it is focusing on supporting the participating children and adolescents to verbalize and express thoughts and emotions related to their crises, and to share their feelings and emotions in a group structure, aims to cover most of mentioned mechanisms and tools in post-disaster psychotherapy research.

Although preventive medicine is one of the most important field of medicine, preventive mental health interventions have not been researched extensively for children under risk of psychopathology [21]. Studies on how to prevent children under risk showed possible positive effects [17]. Gilham et al. [20], suggesting that cognitive and social skills that will protect children under risk against development of psychiatric disorders could be taught [20]. In a study which designed to prevent development of later depression among risky children, Gilham et al. [20] pointed out substantial positive results that led to less depressive symptoms among children two years after attending the program. Numerous other prevention programs have also shown decrease in psychological symptoms, and maintenance of the gains was preserved following early intervention programs $[15,23,35]$. However most of these studies were evaluating only depressive symptoms $[15,35,36]$. While our findings point out global symptom decrease two year after the intervention. Our study concerning child survivors of war and displacement, who have showed to be at a higher risk for developing PTSD and Depression [2,12], revealed potential positive effects of CIPCA two year after the CIPCA intervention. It has been proposed that children who have higher risk may be more positively effected by preventing programs $[15,20]$.

\section{Limitations}

Although our study findings point out probable prevent effects for development of psychopathology after traumatic exposure, several limitations hinder to generalise the results. First of all the low sample size limiting our findings. The second limitation is gathering information about children from only one source also may have affected the quality of our results. Furthermore, possible cofounders or bias factors during th two years of time can not be excluded despite the cluster randomization to a control group. However, it is important to state that this is a test of a new intervention which needs further systematic investigations by independent researchers and among different populations to gain scientific evidence.

\section{Conclusion}

Aiming to prevent PTSD and other posttraumatic psychopathology among displaced child survivors of war, a single session of CIPCA has presumed to be effective in preventing and/ or relieving the symptoms two year after the intervention. The findings point out the possible preventing effect of CIPCA among children at risk. The results of both one-year and two-year follow up in Iraqi Kurdistan are promising and need further evidence. However, the effectiveness of the intervention must be investigate more systemically in later studies before proposing the CIPCA intervention for children in post-disaster situations.

\section{Acknowledgement}

None.

\section{Conflict of Interest}

None.

\section{References}

1. UNHCR (2016) UNHCR Global Trends 2015.

2. Attanayake V, McKay R, Joffres M, Singh S, Burkle F, et al. (2009) Prevalence of mental disorders among children exposed to war: a systematic review of 7,920 children. Med Confl Surviv 25(1): 4-19.

3. Kar N (2009) Psychological impact of disasters on children: review of assessment and interventions. World journal of pediatrics 5(1): 5-11.

4. Nasiroğlu S, Ceri V (2016) Posttraumatic stress and depression in Yazidi refugees. Neuropsychiatric disease and treatment 12: 29412948.

5. Özer S, Şirin S, Oppedal B (2013) Bahçeşehir study of Syrian refugee children in Turkey.

6. Alun McDonald MB, Sonia Khush, Marcia Brophy (2017) Invisible Wounds, The Impact of Six Years of War on The Mental Health of Syria's Children, UK.

7. Fazel M, Reed R, Stein A (2015) Refugee, asylum-seeking and internally displaced children and adolescents. In: Thapar D et al. (Eds.), Rutter's Child and Adolescent Psychiatry, John Wiley \& Sons, Ltd., USA, pp. 573-585.

8. Hodes M (2008) Psychopathology in refugee and asylum seeking children. In: Michael DB, et al. (Eds.), Rutter's Child and Adolescent Psychiatry, Wiley, USA, pp. 474-486.

9. Ahmad A (1992) Symptoms of posttraumatic stress disorder among displaced Kurdish children in Iraq-victims of a man-made disaster after the Gulf war. Nordic Journal of Psychiatry 46(5): 315-319.

10. Dyregrov A, Gupta L, Gjestad R, Mukanoheli E (2000) Trauma exposure and psychological reactions to genocide among Rwandan children. Journal of Traumatic Stress 13(1): 3-21.

11. Heptinstall E, Sethna V, Taylor E (2004) PTSD and depression in refugee children. European child \& adolescent psychiatry 13(6): 373-380.

12. Fazel M, Wheeler J, Danesh J (2005) Prevalence of serious mental disorder in 7000 refugees resettled in western countries: a systematic review. The Lancet 365(9467): 1309-1314.

13. Rose SC, Bisson J, Churchill R, Wessely S (2002) Psychological debriefing for preventing post traumatic stress disorder (PTSD). Cochrane Database Syst Rev 2: CD000560.

14. Bonanni P (1999) Demographic impact of vaccination: a review. Vaccine 17 Suppl 3: S120-S125.

15. Jaycox LH, Reivich KJ, Gillham J, Seligman ME (1994) Prevention of depressive symptoms in school children. Behav Res Ther 32(8): 801816.

16. Albee GW, Gullotta TP (1986) Facts and fallacies about primary prevention. The journal of primary prevention 6(4): 207-218.

17. Albee GW (1980) The fourth mental health revolution. The Journal of prevention 1(2): 67-70.

18. Ahmad A (2014) Crisis intervention program for children and adolescents (CIPCA) to prevent posttraumatic psychopathology, preliminary report. Duhok Medical Journal 8(2): 1-11.

19. Ahmad A (2015) A crisis intervention program to prevent posttraumatic psychopathology among war displaced children in Iraqi Kurdistan. IACAPAP Bulletin 42: 7-9. 
20. Gillham JE, Reivich KJ, Jaycox LH, Seligman ME (1995) Prevention of depressive symptoms in schoolchildren: Two-year follow-up. Psychological science 6(6): 343-351.

21. Fazel M, Betancourt TS (2017) Preventive mental health interventions for refugee children and adolescents in high-income settings. The Lancet Child \& Adolescent Health 2(2): 121-132.

22. Hodgkinson PE, Stewart M (1991) Coping with catastrophe: A handbook of disaster management. Taylor \& Frances/Routledge, USA, pp. 240.

23. Austin LS, Godleski LS (1999) Therapeutic approaches for survivors of disaster. Psychiatric Clinics 22(4): 897-910.

24. Pfefferbaum B, Newman E, Nelson SD (2014) Mental health interventions for children exposed to disasters and terrorism. Journal of child and adolescent psychopharmacology 24(1): 24-31.

25. Buckley TC, Blanchard EB, Neill, WT (2000) Information processing and PTSD: A review of the empirical literature. Clin Psychol Rev 20(8): 1041-1065.

26. Lecic-Tosevski D, Draganic-Gajic S, Pejovic-Milovancevic M, PopovicDeusic S, Christodoulou N, et al. (2014) Child is father of the man: child abuse and development of future psychopathology. Psychiatriki 25(3): 185-191.

27. Levy KN (2013) Introduction: Attachment theory and psychotherapy. Journal of clinical psychology 69(11): 1133-1135.

28. Benz C, Bull T, Mittelmark M, Vaandrager L (2014) Culture in salutogenesis: the scholarship of Aaron Antonovsky. Global Health Promot 21(4): 16-23.
29. Sattler DN, Boyd B, Kirsch J (2014) Trauma-exposed firefighters: Relationships among posttraumatic growth, posttraumatic stress, resource availability, coping and critical incident stress debriefing experience. Stress and Health 30(5): 356-365.

30. Ahmad A, Abdul-Majeed AM, Siddiq AA, Jabar F, Qahar J, et al. (2007) Reporting Questionnaire for Children as a screening instrument for child mental health problems in Iraqi Kurdistan. Transcultural psychiatry 44(1): 5-26.

31. Ahmad A, Sundelin-Wahlsten V, Sofi M, Qahar J, Von Knorring AL (2000) Reliability and validity of a child-specific cross-cultural instrument for assessing posttraumatic stress disorder. European child \& adolescent psychiatry 9(4): 285-294.

32. Ahmad A, Mohamad K (1996) The socioemotional development of orphans in orphanages and traditional foster care in Iraqi Kurdistan. Child Abuse \& Neglect 20(12): 1161-1173.

33. Achenbach TM, Edelbrock C (1991) Child behavior checklist. University of Vermont Department of Psychiatry, USA.

34. Coates S, Schechter D (2004) Preschoolers' traumatic stress post9/11: relational and developmental perspectives. Psychiatric Clinics 27(3): 473-489.

35. Stark KD, Reynolds WM, Kaslow NJ (1987) A comparison of the relative efficacy of self-control therapy and a behavioral problemsolving therapy for depression in children. J Abnorm Child Psychol 15(1): 91-113.

36. Kahn JS (1990) Comparison of Cognitive-Behavioral, Relaxation, and Self-Modeling Interventions for Depression among Middle-School Students. School Psychology Review 19(2): 196-211. 